\title{
Incorporating genetic counseling into clinical care for children and adolescents with cancer
}


Jessica N Everett ${ }^{*, 1,2}$, Rajen J Mody ${ }^{2,3}$, Elena M Stoffel' \& Arul M Chinnaiyann ${ }^{2,45,5,6}$

First draft submitted: 17 November 2015; Accepted for publication: 2 February 2016; Published online: 18 February 2016

Genetic counseling and genetic testing is becoming an increasingly key component of multidisciplinary cancer care, as evidenced by inclusion of cancer risk assessment and genetic counseling in the clinical service standards of professional societies and accrediting bodies (e.g., The American College of Surgeons Commission on Cancer [1] and the American Society of Clinical Oncology [2]). Identification of patients at risk for hereditary cancer syndromes can provide opportunities to optimize care for the existing cancer, and guide surveillance for cancer survivors who may be at risk for second (or third) malignancies. Identifying patients with hereditary cancer syndromes also benefits their family members, who can then engage in cancer prevention strategies to improve outcomes. There are several known hereditary cancer syndromes that can present with childhood tumors, including Li-Fraumeni syndrome, hereditary paraganglioma, Von Hippel Lindau syndrome and others, and pediatric oncology patients and their families can benefit from incorporating genetic services into existing care teams.

With increasing use of next-generation DNA sequencing (NGS) technology in clinical diagnostic testing, pathogenic variants in cancer risk genes are being identified as secondary or incidental findings in children and adolescents who have sequencing completed for other reasons. In the first 104 patients with cancer enrolled in our Peds-MiOncoSeq population, 91 completed sequencing of paired tumor/normal DNA samples, and nine $(10 \%)$ had pathogenic germline variants associated with cancer risk [3]. The majority of the pathogenic germline variant findings (7/9) were not known prior to study participation and $4 / 9$ had no family history to suggest potential risk for a familial

\section{KEYWORDS}

- genetic counseling • genetic testing $\bullet$ pediatric oncology - precision oncology $\bullet$ tumor sequencing

\section{"Genetic counseling and genetic testing is becoming an increasingly key component of multidisciplinary cancer care...”}


“...identifying children and adolescents at risk for hereditary cancer syndromes creates opportunities to optimize cancer treatment and long-term surveillance.” cancer syndrome. Similarly, the Pediatric Cancer Genome Project found germline mutations in $8.5 \%$ of pediatric oncology patients completing genome or exome sequencing, and $60 \%$ of those with available family history information showed no evidence of inherited risk [4]. Even if sequencing is not performed on paired normal DNA, tumor sequencing can lead to identification of germline mutations [5], or identify mutational profiles strongly suggestive of underlying inherited risk, as with the ultra-hypermutant tumors seen in children with bi-allelic mismatch repair deficiency syndrome [6]. Molecular testing of germline DNA is frequently ordered in the diagnostic evaluation of cancer-free children with developmental delay, congenital malformations and/or dysmorphic features, and can lead to secondary findings in cancer risk genes as well. One large study of array comparative genomic hybridization found that $0.34 \%$ of total results and $1.2 \%$ of abnormal results had a clearly cancer predisposing copy number variant [7]. Exome sequencing is taking precedence as a diagnostic test in pediatric patients with unexplained developmental delay or syndromic features, and a study of 2000 patients ( $88 \%$ pediatric) referred for clinical exome sequencing similarly found $1.25 \%$ had an incidental finding in a cancer predisposition gene $[8]$, including those recommended for reporting by the American College of Medical Genetics [9].

The increased use of NGS in various clinical settings along with the potential implications for long-term follow-up for pediatric patients and family members affected by inherited cancer syndromes raises the important question of how to best meet the clinical need for timely genetic evaluation of these patients. Incorporating genetics services into pediatric oncology care teams offers several advantages, and there are some key steps that can help accomplish this goal.

\section{Step 1: identifying patients who may benefit from genetic services}

About $5-10 \%$ of cancer diagnoses in general are estimated to be caused by inherited risk. This number can be substantially higher for certain tumor types, including some cancers that occur in children or adolescents [10]. A single study of a childhood cancer survivor clinic evaluated 370 patients for criteria suggestive of inherited risk, and found that $29 \%$ could potentially benefit from genetic evaluation [11]. Among patients identified through this screening, 34\% were identified based on personal history of a cancer diagnosis with known association to an underlying syndrome or clinical features of a syndrome. Referral for genetic evaluation has been suggested for individuals with specific tumor types [10], and it is likely that this list will grow over time. Although family history of cancer is frequently employed as a screen for hereditary syndromes, it is also worth noting that not all pediatric patients with genetic predisposition to cancer will have a family history of related cancers. Small family size, young ages of relatives, variable penetrance and de novo mutations can make family history unreliable as a primary screening tool. Nearly half of Peds-MiOncoSeq participants with germline mutations in cancer risk genes had no suggestive family history [3], emphasizing the importance of looking beyond the family history to recognize tumor types that suggest genetic risk.

\section{Step 2: collecting \& updating family histories}

In the previously mentioned survivor clinic screening study, $66 \%$ of patients who could benefit from genetic evaluation were identified on the basis of reported family histories of cancer or other genetic conditions [11], emphasizing the importance of comprehensive family history collection. An American Society of Clinical Oncology expert statement recommends minimum elements for family history collection in the oncology setting, which includes: type of primary cancer and age at diagnosis for all firstand second-degree relatives (parents, siblings, children, aunts, uncles and grandparents), ethnicity and results of any previous genetic testing in the family [12]. Importantly, family histories are dynamic and new diagnoses of cancer within the family over time may become relevant in assessing risk. This is particularly important in the pediatric setting, where parents, aunts and uncles may be quite young, and may develop related cancers after the diagnosis in a child. For this reason, family histories must be updated as childhood cancer survivors are followed over time. Optimal collection and updating of family history could be improved with better tools that can be integrated into existing practice, particularly if the minimum recommended elements could be integrated into electronic health record systems. Options for direct patient data entry, and/or auto prompts for genetic counseling referral, could also streamline this process 
and improve identification of patients who may benefit from genetic evaluation.

\section{Step 3: including genetic counselors as part of the multidisciplinary team}

There are many potential advantages to incorporating genetic counseling services directly into the multidisciplinary care team for children and adolescents with cancer. The nature and complexity of cancer care can already mean multiple appointments with specialists on different days, creating barriers for children and their families/ caregivers including more missed days of school or work, difficulties navigating a health system to correctly schedule visits and even transportation needs. One study of outcomes in a multidisciplinary pediatric cancer survivors' clinic with same-day coordination of visits found that $26 / 130$ participants (20\%) had failed to followup on a specialty referral in the past, and were able to complete these evaluations when they were offered as a same-day service [13].

Direct involvement of a genetic counselor in the pediatric oncology clinic may also improve patient identification. Adding a genetic counselor to screen patients for suggestive tumor types and family histories in a pediatric survivors' clinic led to a significant increase in patient identification, from $6 \%$ referred prior to $29 \%$ after addition of genetic counselor [11].

While there are more than 4000 trained genetic counselors in USA [14], with about 30\% identifying cancer genetics as their primary specialty (2014 National Society of Genetic Counselors Professional Status Survey [15]), not all clinics will have easy access to an on-site genetic counselor. Genetic counselors can be located by geographic area using the National Society of Genetic Counselors Find a Counselor feature [15] and clinics can also be located through the National Cancer Institute's Cancer Genetics Services Directory [16]. There are also increasing options for phone or video counseling to help meet demand for services in underserved areas. The American Society of Clinical Oncology expert statement on family history collection recommends that all oncology teams should identify providers within or outside their practice with cancer genetics expertise who can provide appropriate counseling [17].

In summary, identifying children and adolescents at risk for hereditary cancer syndromes creates opportunities to optimize cancer treatment and long-term surveillance. A multidisciplinary approach that incorporates genetic counseling services into the existing pediatric oncology team can increase patient identification, reduce barriers to services and enhance patient-centered care.

\section{Financial \& competing interests disclosure}

This work was supported by grant funding from the NIHNHGRI Clinical Sequencing Exploratory Research (CSER) Consortium 1UM1HG006508. The authors have no other relevant affiliations or financial involvement with any organization or entity with a financial interest in or financial conflict with the subject matter or materials discussed in the manuscript apart from those disclosed.

No writing assistance was utilized in the production of this manuscript.

\section{References}

1 Commission on Cancer Cancer Program Standards 2012: Ensuring Patient-Centered Care.

www.facs.org

2 Robson ME, Bradbury AR, Arun B et al. American Society of Clinical Oncology Policy Statement Update: genetic and genomic testing for cancer susceptibility. J. Clin. Oncol. 33(31), 3660-3667 (2015).

3 Mody RJ, Wu YM, Lonigro RJ et al. Integrative clinical sequencing in the management of refractory or relapsed cancer in youth. JAMA 314(9), 913-925 (2015).

4 Zhang J, Walsh MF, Wu G et al. Germline mutations in predisposition genes in pediatric cancer. N. Engl. J. Med. 373(24), 2336-2346 (2015).
5 Bombard Y, Robson M, Offit K. Revealing the incidentalome when targeting the tumor genome. JAMA 310 (8), 795-796 (2013).

6 Shlien A, Campbell BB, De Borja R et al. Combined hereditary and somatic mutations of replication error repair genes result in rapid onset of ultra-hypermutated cancers. Nat. Genet. 47(3), 257-262 (2015).

7 Hamm JA, Mikhail FM, Hollenbeck D, Farmer M, Robin NH. Incidental detection of cancer predisposition gene copy number variations by array comparative genomic hybridization. J. Pediatr. 165(5), 1057-1059.e1-4 (2014).

8 Yang Y, Muzny DM, Xia F et al. Molecular findings among patients referred for clinical whole-exome sequencing. JAMA 312(18), 1870-1879 (2014).
9 Green RC, Berg JS, Grody WW et al. ACMG recommendations for reporting of incidental findings in clinical exome and genome sequencing. Genet. Med. 15(7), 565-574 (2013).

10 Knapke S, Zelley K, Nichols KE, Kohlmann W, Schiffman JD. Identification, management, and evaluation of children with cancer-predisposition syndromes. Presented at: 2012 ASCO Annual Meeting. Chicago, IL, USA, 1-5 June 2012.

11 Knapke S, Nagarajan R, Correll J, Kent D, Burns K. Hereditary cancer risk assessment in a pediatric oncology follow-up clinic. Pediatr. Blood Cancer 58(1), 85-89 (2012).

$12 \mathrm{Lu} \mathrm{KH}$, Wood ME, Daniels M et al. American society of clinical oncology expert statement: collection and use of a cancer 
EDITORIAL Everett, Mody, Stoffel \& Chinnaiyan

family history for oncology providers. J. Clin. Oncol. 32(8), 833-840 (2014).

13 Carlson CA, Hobbie WL, Brogna M, Ginsberg JP. A multidisciplinary model of care for childhood cancer survivors with complex medical needs. J. Pediatr. Oncol. Nurs. 25(1), 7-13 (2008).
14 American Board of Genetic Counseling. www.abgc.net

15 National Society of Genetic Counselors. http://nsgc.org/

16 National Cancer Institute Cancer Genetics Services Directory.

www.cancer.gov
$17 \mathrm{Lu} \mathrm{KH}$, Wood ME, Daniels M et al. American Society of Clinical Oncology Expert Statement: collection and use of a cancer family history for oncology providers. J. Clin. Oncol. 32(8), 833-840 (2014). 